\title{
Multivariate allometric comparisons for kuruma shrimp (Penaeus japonicus) off Taiwan
}

\author{
Tzong-Der Tzeng ${ }^{\mathrm{a}, \mathrm{b}}$, Shean-Ya Yeh ${ }^{\mathrm{b}, *}$ \\ ${ }^{a}$ College of Liberal Education, Shu-Te University, Kaohsiung County 824, Taiwan \\ ${ }^{\mathrm{b}}$ Institute of Oceanography, National Taiwan University, P.O. Box 23-13, Taipei 106, Taiwan
}

Received 19 February 2001; accepted 31 October 2001

\begin{abstract}
Multivariate allometric coefficients for kuruma shrimp (Penaeus japonicus) in five geographic locations (N27, N24, TAS, PUT, and TON) off Taiwan were estimated and compared by sex. Eight morphometric measurements were made for each individual. The 1st eigenvector obtained from the covariance matrix of log-transformed data was used to represent the multivariate allometric coefficients. The bootstrap method was used to assess the accuracy of estimates. A dendrogram of five sampling areas was constructed by unweighted pair-group method with arithmetic means (UPGMA) using the angles between five 1st eigenvectors, and a permutation test was used to test if the difference between two multivariate allometric coefficients obtained from different populations/groups was significant. Five areas were clustered into two distinct groups for each sex, the first group included N27, N24, and TAS; the second one included TON and PUT. For each sex, permutation tests indicated that the difference of each within group pairwised multivariate allometric coefficients is not significant and of each between groups pairwised multivariate allometric coefficients is significant. Because (1) five populations considered here are from the same stock and (2) the size of individuals in the second group is smaller than those in the first group, we concluded that the allometry in life stages differs for this species. The difference between multivariate allometric coefficients in both sexes was also tested based on the two second group data sets, and the result showed that the difference between sexes is significant.
\end{abstract}

(C) 2002 Elsevier Science B.V. All rights reserved.

Keywords: Penaeus japonicus; Allometry; Bootstrap; Angle; Permutation test

\section{Introduction}

Allometric analyses can assess the covariation of characters (Cock, 1966), and provide a method to elucidate the relationship between processes of growth and evolution (Blackstone, 1987). Morphometric allometric relationships have been developed for bivariate allometric equation and for a multivariate generalization of the bivariate allometric equation.

\footnotetext{
* Corresponding author. Tel.: +886-2-23637753; fax: +886-2-23661197.

E-mail address: tdertzeng@kimo.com.tw (S.-Y. Yeh).
}

The formula of bivariate allometry (Huxley, 1932) assumes a power function of the form $y=b x^{\alpha}$, where $x$ and $y$ are measurements and the constant $\alpha$ is often called the allometric coefficient. The special case when $\alpha=1$ is called isometry. Jolicoeur (1963) used the 1 st eigenvector extracted from the covariance matrix of log-transformed data to reflect the multivariate allometric coefficients. When all loadings of the 1 st eigenvector are equal with a value 1 divided by the square root of the number of variable, the 1 st eigenvector is called the isometry vector (Jolicoeur, 1963). The multivariate allometric coefficients can be easily translated to bivariate allometric coefficients by 
using the ratio of the coefficients in the 1st eigenvector for two variables corresponding to variable in the bivariate allometric analysis (Shea, 1985).

Kuruma shrimp (Penaeus japonicus) is one of the most abundant and highly valued species in Taiwan trawl fisheries in the Taiwan Strait and the East China Sea. There are many studies on the biology of this species in this area (Liao and Chen, 1972; Tsai et al., 1992; Tzeng and Yeh, 1999a), but there is no information on allometry. The aim of this study is to estimate and compare the multivariate allometric coefficients in the kuruma shrimp in five areas off Taiwan.

\section{Materials and methods}

Five samples were collected from five adjacent waters (N27, N24, TAS, PUT, and TON) off Taiwan during December in 1995 (Fig. 1). Sex was identified and separated. Eight morphometric measurements were made on each specimen (Fig. 2). They were antennal spine width (ASW), hepatic spine width (HSW), carapace length (CL), diagonal carapace length (DCL), 1st abdominal segment length (FSL), 1st abdominal segment width (FSW), 1st abdominal segment height (FSH), and body length (BL). The sample size, sampling area code, mean and range of carapace length are summarized in Table 1. The average sizes of individuals in PUT and TON were smaller than those in other areas.

Estimation of multivariate allometric coefficients followed the recommendation of Jolicoeur (1963). The 1st eigenvector extracted from the covariance matrix of log-transformed data for each sampling area and sex was used to represent the multivariate allometric coefficients. A bootstrap technique (Efron and Tibshirani, 1986) was used to assess the accuracy of allometric coefficients. A bootstrap sample of

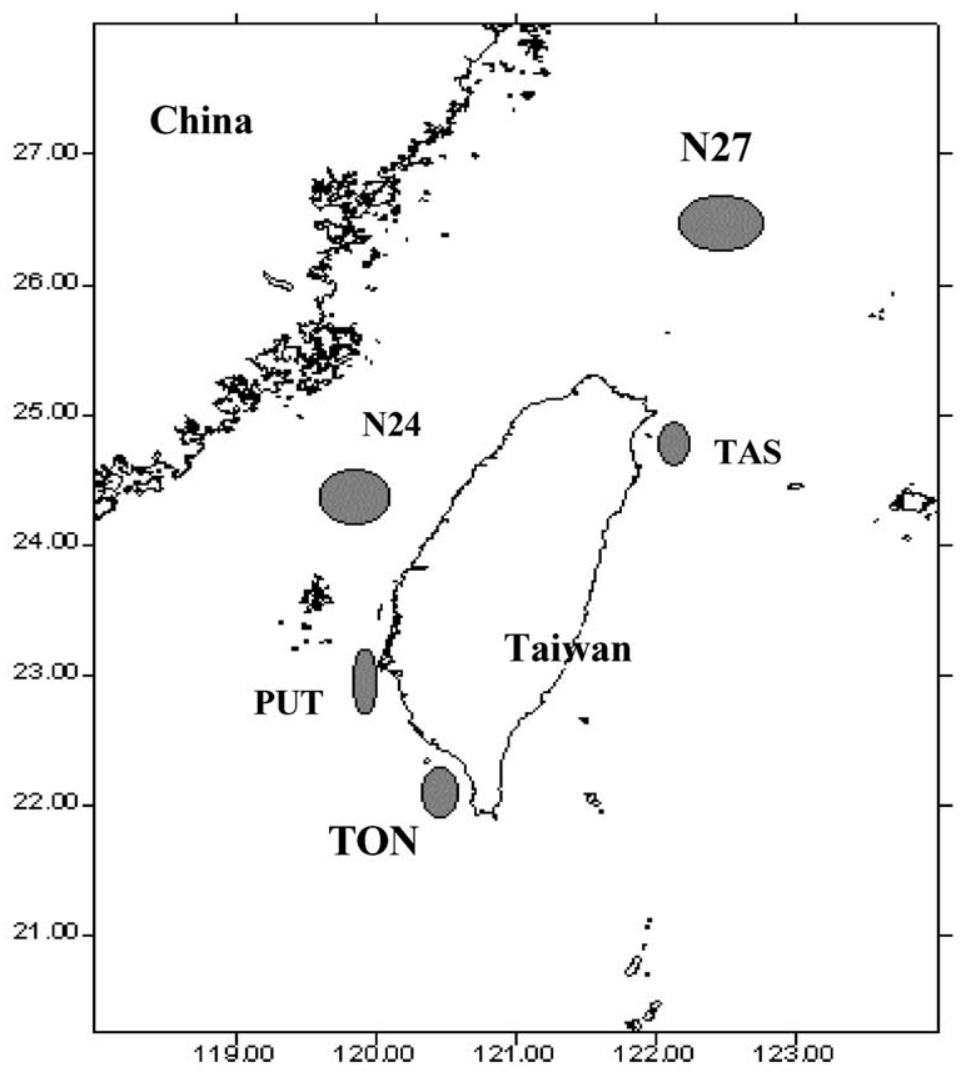

Fig. 1. The sampling areas off Taiwan. 


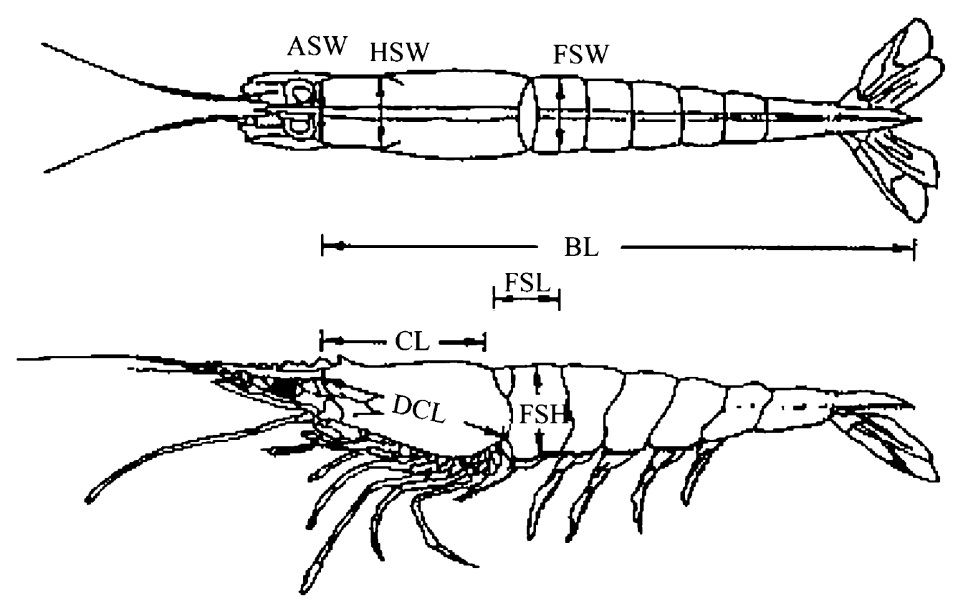

Fig. 2. Diagram of Penaeus japoncus showing the body parts measured.

corresponding sample size was drawn at random, with replacement. For each analysis, 5000 bootstrap iterations were performed.

The angles between five 1st eigenvectors indicate their similarity in multivariate allometric coefficients obtained from different geographic populations. The angle $\alpha$ was computed as the arc cosine of the inner product of the two 1st eigenvectors (Gibson et al., 1984), $\alpha=\arccos (b c) \times 180 / \pi$, where $b$ and $c$ are the 1 st eigenvectors. If the angle increases, the similarity decreases. These angles were considered as distance coefficients, and then used in cluster analysis. The dendrogram was constructed by unweighted pairgroup method with arithmetic means (UPGMA) by using these angles by sex. The angles between iso- metric vector and these 1st eigenvectors were also computed, respectively.

Permutation test poposed by Tzeng and Yeh (1999b) was used to test if the difference between two multivariate allometric coefficients is significant. The angle between two multivariate allometric coefficients was taken as test statistic. For each permutation test, 5000 iterations were performed. The position of the angle $\left(\theta_{0}\right)$ estimated obtained from original data sets was determined among the ordered values of the angles $\left(\theta_{1}\right)$ obtained from permuted data sets. The proportion $(P)$ of the observed angle values greater than or equal to $\theta_{0}$ was also calculated. This $P$ value can be interpreted in the same way as for conventional tests of significance: if it is less than $5 \%$ then this

Table 1

Code of sampling area, sex, sample size and mean, standard deviation (S.D.), and range of carapace length (CL, mm) of kuruma shrimp in the adjacent waters off Taiwan collected during December 1995

\begin{tabular}{|c|c|c|c|c|c|c|}
\hline Area code & Sex & Sampling site & Sample size & Mean of CL & S.D. of CL & Range of CL \\
\hline \multirow[t]{2}{*}{ N27 } & $\mathrm{F}$ & $26-27^{\circ} \mathrm{N}$ & 101 & 58.20 & 2.07 & $55.02-62.84$ \\
\hline & M & $122-123^{\circ} \mathrm{E}$ & 122 & 48.19 & 2.16 & $43.93-54.42$ \\
\hline \multirow[t]{2}{*}{ N24 } & $\mathrm{F}$ & $24^{\circ} 10^{\prime}-24^{\circ} 15^{\prime} \mathrm{N}$ & 54 & 59.97 & 2.90 & $55.61-65.64$ \\
\hline & M & $119^{\circ} 36^{\prime}-120^{\circ} \mathrm{E}$ & 79 & 45.73 & 2.64 & $42.01-55.58$ \\
\hline \multirow[t]{2}{*}{ TAS } & $\mathrm{F}$ & Water off & 38 & 59.76 & 2.41 & $55.47-65.03$ \\
\hline & M & Tashi harbor & 44 & 48.03 & 2.88 & $43.34-55.78$ \\
\hline \multirow[t]{2}{*}{ PUT } & $\mathrm{F}$ & Water off & 59 & 35.30 & 2.95 & $31.18-40.69$ \\
\hline & M & Putai harbor & 58 & 35.14 & 2.68 & $31.20-40.40$ \\
\hline \multirow[t]{2}{*}{ TON } & $\mathrm{F}$ & Water off & 102 & 34.75 & 2.78 & $31.19-40.64$ \\
\hline & M & Tonkong harbor & 66 & 34.22 & 2.68 & $31.18-40.38$ \\
\hline
\end{tabular}


provides some evidence that the null hypothesis is not true, if it is less than $1 \%$ then it provides strong evidence that the null hypothesis is not true (Tzeng and Yeh, 1999b).

\section{Results}

Multivariate allometric coefficients and the percentages of total variance explained by the 1 st eigenvalues are shown in Table 2. All the 1st eigenvalues explained the largest part of total variance. For females, the maximum percentage was $96.03 \%$ estimated from TAS, but the smallest one was $86.44 \%$ estimated from the N27. The largest and smallest percentages of total variance explained by the 1st eigenvalues for males were separate from TAS and N27, ranging from 97.93 to $96.54 \%$. All 1st eigenvectors have all-positive and near-equal loadings and are interpreted as representing multivariate allometric coefficients. The estimates of multivariate allometric coefficients were considered fairly stable, as indicted by the relatively small standard errors of the estimates.

The theoretical value for isometric coefficient $(\alpha)$ was 0.3535 . The angles between these 1 st eigenvectors and isometric vector are also shown in Table 2 for both sexes. The angle values range from 3.8106 to $3.1427^{\circ}$ for females, and from 4.3514 to $2.7904^{\circ}$ for males. The multivariate allometric coefficients estimated from TON in females and from N24 in males deviated most from the isometric vector. Allometric coefficients for some characters were clearly different from isometry value (Table 2 ).

The angles between five multivariate allometric coefficients for females and males are shown in Table 3. For females, the angle $\left(4.8670^{\circ}\right)$ between multivariate allometric coefficients obtained from $\mathrm{N} 24$ and TON data sets was least similar, but the one $\left(1.6909^{\circ}\right)$ obtained from data sets of N27 and TAS was most similar. The angles between five multivariate allometric coefficients ranged from 3.6035 to $1.4214^{\circ}$ for males. Two multivariate allometric coefficients estimated from N24 and TON data sets had the smallest similarity, but two estimated from data sets of PUT and TON had the highest similarity.

Fig. 3 shows the dendrograms of five sampling areas for females and males. Two results were very similar. Five sampling areas were clustered into two distinct groups, the first group included N27, N24 and TAS and the second group included PUT and TON. The results of permutation test for both sex indicated that pairwise multivariate allometric coefficients for the populations in the same group are not different, but pairwise multivariate allometric coefficients for the populations in different groups are significantly different (Table 3).

Thus the specimens in the same group were pooled, and then multivariate allometric coefficient was reestimated in each group. All 1st eigenvectors have

\section{Female}

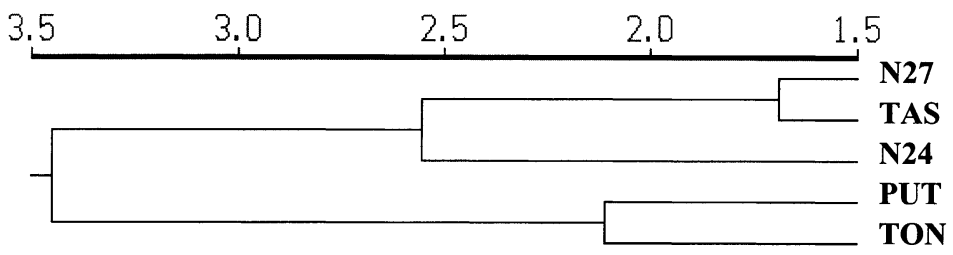

\section{Male}

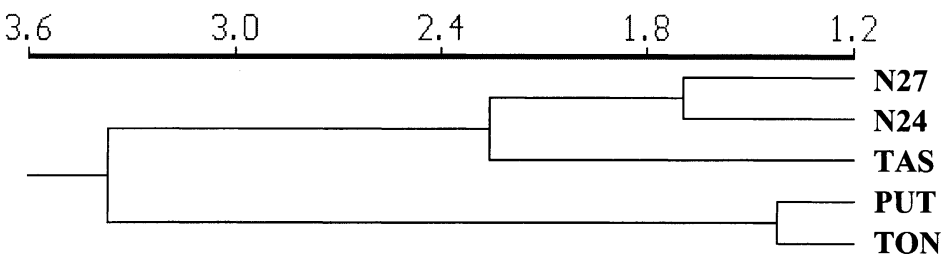

Fig. 3. Dendrograms of five sampling areas for female (above) and males (below). 
Table 2

Allometric coefficients with corresponding S.D. (in parentheses), percentages of total variance (\% variance) explained by the 1 st eigenvalues and the angle $\left(\theta\left(^{\circ}\right)\right.$ ) between multivariate allometric coefficients and isometric vector for females and males kuruma shrimp from five sampling areas

\begin{tabular}{|c|c|c|c|c|c|c|c|c|c|c|}
\hline \multirow[t]{2}{*}{ Area } & \multicolumn{8}{|l|}{ Variable } & \multirow[t]{2}{*}{$\%$ Variance } & \multirow[t]{2}{*}{$\theta\left(^{\circ}\right)$} \\
\hline & ASW & HSW & $\mathrm{CL}$ & DCL & FSL & FSW & FSH & BL & & \\
\hline \multicolumn{11}{|c|}{ Female } \\
\hline N27 & $0.3463(0.0146)$ & $0.3872(0.0076)$ & $0.3418(0.0129)$ & $0.3444(0.0103)$ & $0.3741(0.0139)$ & $0.3321(0.0227)$ & $0.3624(0.0169)$ & $0.3360(0.0070)$ & 86.44 & 3.2516 \\
\hline $\mathrm{N} 24$ & $0.3696(0.0102)$ & $0.3716(0.0105)$ & $0.3641(0.0134)$ & $0.3428(0.0118)$ & $0.3797(0.0105)$ & $0.3251(0.0150)$ & $0.3448(0.0178)$ & $0.3258(0.0061)$ & 94.96 & 3.5257 \\
\hline TAS & $0.3528(0.0086)$ & $0.3894(0.0080)$ & $0.3496(0.0091)$ & $0.3584(0.0103)$ & $0.3510(0.0116)$ & $0.3314(0.0090)$ & $0.3612(0.0204)$ & $0.3308(0.0048)$ & 96.03 & 3.1427 \\
\hline PUT & $0.3508(0.0119)$ & $0.3686(0.0184)$ & $0.3386(0.0090)$ & $0.3425(0.0091)$ & $0.3502(0.0091)$ & $0.3548(0.0136)$ & $0.3933(0.0151)$ & $0.3250(0.0094)$ & 93.80 & 3.4255 \\
\hline TON & $0.3226(0.0101)$ & $0.3859(0.0101)$ & $0.3425(0.0073)$ & $0.3492(0.0076)$ & $0.3437(0.0109)$ & $0.3616(0.0139)$ & $0.3852(0.0114)$ & $0.3319(0.0064)$ & 92.39 & 3.8106 \\
\hline \multicolumn{11}{|l|}{ Male } \\
\hline $\mathrm{N} 27$ & $0.3273(0.0041)$ & $0.3319(0.0070)$ & $0.3457(0.0043)$ & $0.3481(0.0037)$ & $0.3692(0.0066)$ & $0.3672(0.0074)$ & $0.4044(0.0063)$ & $0.3272(0.0025)$ & 96.54 & 4.2706 \\
\hline $\mathrm{N} 24$ & $0.3390(0.0058)$ & $0.3121(0.0115)$ & $0.3397(0.0055)$ & $0.3516(0.0062)$ & $0.3651(0.0091)$ & $0.3805(0.0063)$ & $0.3979(0.0071)$ & $0.3348(0.0025)$ & 97.36 & 4.3514 \\
\hline TAS & $0.3611(0.0059)$ & $0.3185(0.0056)$ & $0.3497(0.0062)$ & $0.3382(0.0050)$ & $0.3479(0.0071)$ & $0.3726(0.0134)$ & $0.4024(0.0069)$ & $0.3308(0.0031)$ & 97.93 & 4.2158 \\
\hline PUT & $0.3625(0.0103)$ & $0.3538(0.0085)$ & $0.3391(0.0055)$ & $0.3415(0.0056)$ & $0.3701(0.0091)$ & $0.3483(0.0098)$ & $0.3788(0.0071)$ & $0.3314(0.0028)$ & 97.42 & 2.7904 \\
\hline TON & $0.3584(0.0065)$ & $0.3458(0.0049)$ & $0.3372(0.0041)$ & $0.3310(0.0043)$ & $0.3881(0.0093)$ & $0.3509(0.0087)$ & $0.3735(0.0073)$ & $0.3393(0.0031)$ & 97.52 & 3.2871 \\
\hline
\end{tabular}


Table 3

The angles $\left({ }^{\circ}\right)$ between five multivariate allometric coefficients in five sampling areas for females and males

\begin{tabular}{llllll}
\hline \multicolumn{5}{l}{ Sampling area } \\
\cline { 2 - 6 } & $\mathrm{N} 27$ & $\mathrm{~N} 24$ & TAS & PUT & TON \\
\hline Female & & & & & \\
N27 & 0.0000 & & & & \\
N24 & 2.4184 & 0.0000 & & & \\
TAS & 1.6909 & 2.6905 & 0.0000 & & \\
PUT & $2.8906^{*}$ & $4.0976^{*}$ & $2.8236^{*}$ & 0.0000 & \\
TON & $3.0944^{*}$ & $4.8670^{*}$ & $2.9264^{*}$ & 2.1118 & 0.0000 \\
Male & & & & & \\
N27 & 0.0000 & & & & \\
N24 & 1.6935 & 0.0000 & & & \\
TAS & 2.5241 & 1.9912 & 0.0000 & & \\
PUT & $3.0516^{*}$ & $3.5475^{*}$ & $3.1458^{*}$ & 0.0000 & \\
TON & $3.2635^{*}$ & $3.6035^{*}$ & $3.6005^{*}$ & 1.4214 & 0.0000 \\
\hline
\end{tabular}

*Value significant at $99 \%$. all-positive and near-equal loadings and are interpreted as representing multivariate allometric coefficients in each group by sex (Table 4). The estimates were considered fairly stable, as indicated by the relatively small standard errors of the estimates.

The difference between multivariate allometric coefficients obtained from two different groups by sex was also tested by permutation test. The frequency distributions of $5000 \theta_{1}$ in females and males was shown in Fig. 4. The $\theta_{0}$ is $4.2573^{\circ}$ in females, and $3.7192^{\circ}$ in males. The value of $P$ is $0 \%$ for each sex. This value is clearly smaller than 1 or $5 \%$, so this test is significant. These indicated that there was significant non-random structure in the data, and supported that the differences between multivariate allometric coefficients from different groups are significant for each sex.
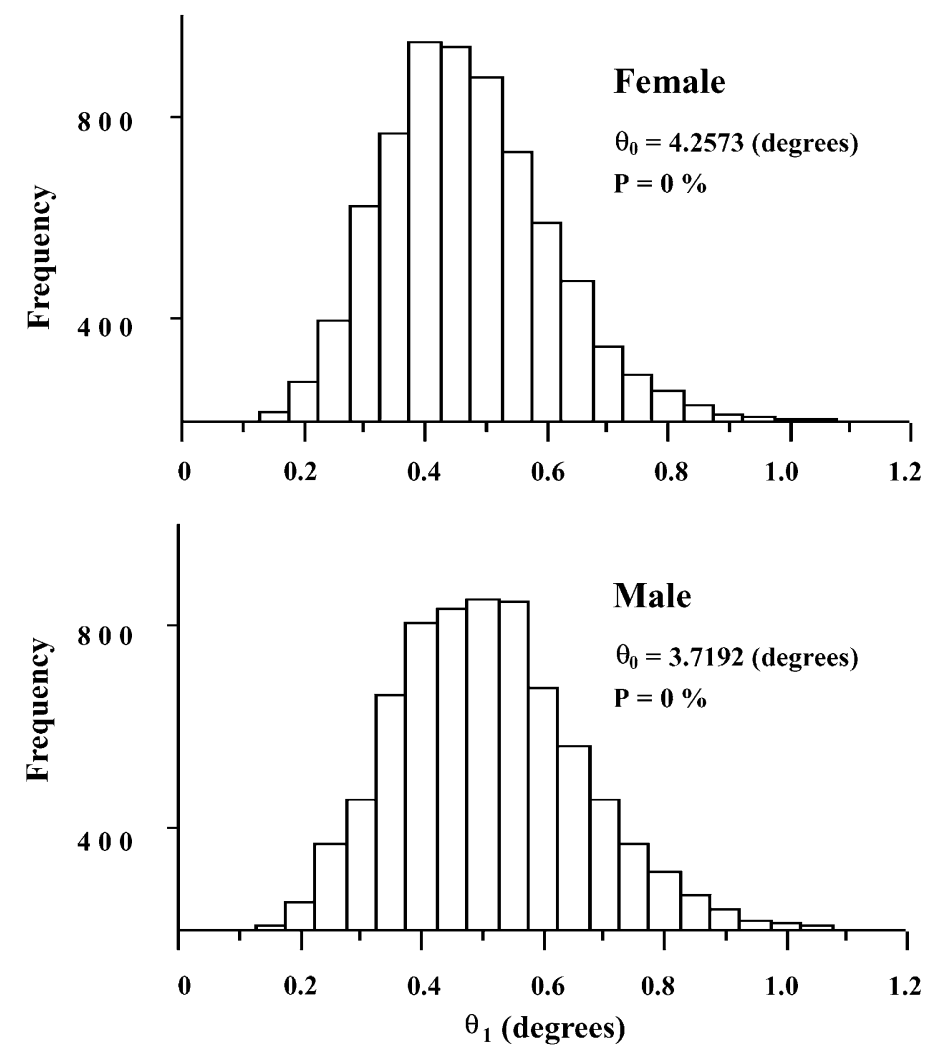

Fig. 4. Frequency distribution of 5000 angles $\left(\theta_{1}\right)$ estimated from the two data sets permuted in females (above) and males (below). $\theta_{0}$ stands for the angle between the two 1st eigenvectors estimated from the original two data sets. $P$ is the proportion of $\theta_{1}$ larger than $\theta_{0}$ among 5000 permutations. 
Table 4

Allometric coefficients with corresponding S.D. (in parentheses), percentages of total variance (\% variance) explained by the 1 st eigenvalues in two different groups for females and males

\begin{tabular}{|c|c|c|c|c|c|c|c|c|c|}
\hline & \multicolumn{8}{|l|}{ Variable } & \multirow[t]{2}{*}{$\%$ Variance } \\
\hline & ASW & HSW & $\mathrm{CL}$ & DCL & FSL & FSW & FSH & $\mathrm{BL}$ & \\
\hline \multicolumn{10}{|l|}{ Female } \\
\hline Group 2 & $0.3307(0.0074)$ & $0.3735(0.0096)$ & $0.3386(0.0059)$ & $0.3455(0.0059)$ & $0.3484(0.0073)$ & $0.3632(0.0101)$ & $0.3869(0.0089)$ & $0.3374(0.0073)$ & 92.48 \\
\hline \multicolumn{10}{|l|}{ Male } \\
\hline Group 1 & $0.3319(0.0030)$ & $0.3177(0.0042)$ & $0.3398(0.0030)$ & $0.3461(0.0024)$ & $0.3647(0.0043)$ & $0.3833(0.0047)$ & $0.4084(0.0037)$ & $0.3267(0.0016)$ & 97.59 \\
\hline Group 2 & $0.3578(0.0055)$ & $0.3484(0.0048)$ & $0.3385(0.0033)$ & $0.3360(0.0033)$ & $0.3801(0.0064)$ & $0.3504(0.0065)$ & $0.3761(0.0051)$ & $0.3379(0.0024)$ & 97.49 \\
\hline
\end{tabular}




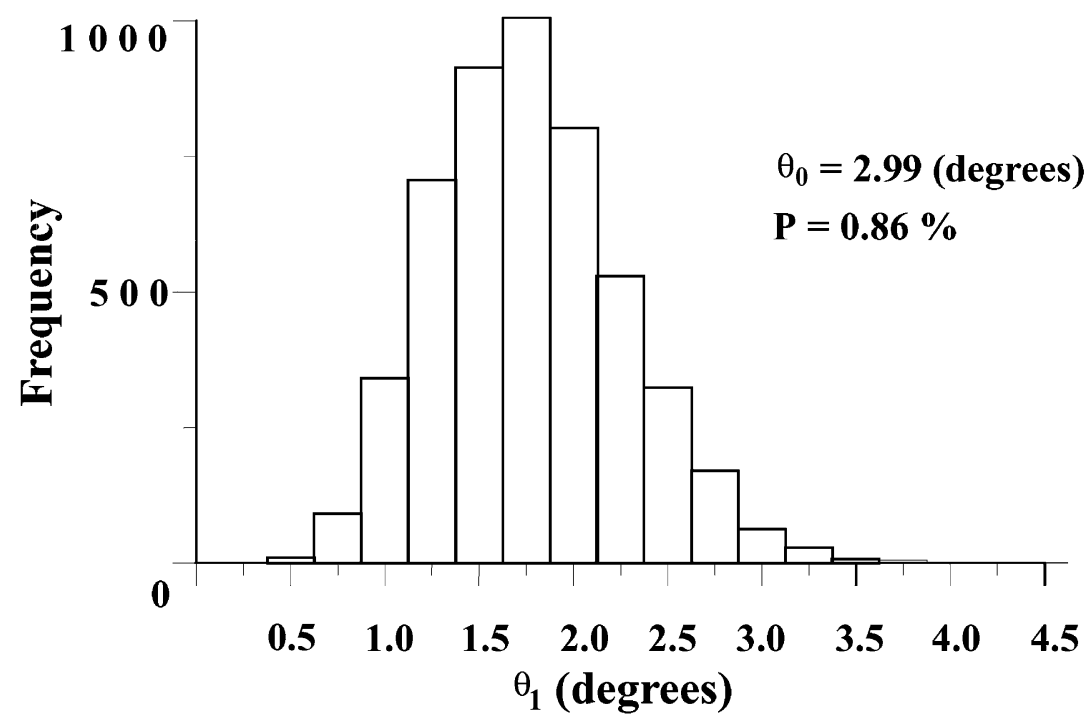

Fig. 5. Frequency distribution of 5000 angles $\left(\theta_{1}\right)$ estimated from the female and male data sets permuted. $\theta_{0}$ stands for the angle between the two 1st eigenvectors estimated from the original two data sets. $P$ is the proportion of $\theta_{1}$ larger than $\theta_{0}$ among 5000 permutations.

To test the difference between multivariate allometric coefficients in both sexes, the individuals in the second groups was first pooled as a data set, and then permutation test was performed based on the two data sets. The histogram of $5000 \theta_{1}$ is shown in Fig. 5. The $\theta_{0}$ value $\left(4.2573^{\circ}\right)$ was extreme in magnitude compared with $5000 \theta_{1}$ resulting from permuted data sets and $P$ value $(0.86 \%)$ is smaller than 1 or $5 \%$. There is thus significant non-random structure in the data, and the allometric difference between both sexes is significant.

\section{Discussion}

Five sampling areas were clustered into two distinct groups, for each sex, suggesting that there might be considerable difference between multivariate allometric coefficients from different geographical populations. The permutation tests indicate it is extremely unlikely that the large angle between multivariate allometric coefficients from original data sets in different groups was due to chance. Therefore, there are two different multivariate allometric patterns in five geographic populations. However, because (1) five populations considered here are from the same stock (Tzeng and Yeh, 1999a), and (2) the size of individuals in the second group is smaller than that in the first group (Table 1), we concluded that allometry in life stages differs for the kuruma shrimp in these waters.

The part of total variance explained by the 1 st eigenvalues ranged from 97.93 to $86.44 \%$ for females and males in this study. The majority of variance in each group was explained by the 1st eigenvalues, which showed a good fit of the model of multivariate allometry to the data in all sampling areas considered here (Bjorklund, 1993). Similar results had been obtained for relative growth in other arthropods (Riska, 1981; Boitard et al., 1982; Tzeng et al., 1998).

Allometry may be modified during growth (Klingenberg, 1996). To elucidate the relationship between populations, the effect of growth variation within/between groups must be decreased (Bookstein et al., 1985; Tzeng et al., 1998). Narrowing the differences of size between groups may reduce such effects. Restricting group comparisons to specific length classes may disregard ontogenetic change within groups, and the information may be necessary for meaningful descriptions of group differences (Bookstein et al., 1985). However, this effect may not be serious in this study, because the carapace length of each individual in each group was not all equal. It is relevant that because biological data are noisy, if the 
range of size included in allometric analysis is too small, the probability is high that the scaling exponent and allometric coefficient will be distorted by sampling error (LaBarbera, 1989).

Allometric analyses can also provide valuable information about evolutionary modifications of growth trajectories and about patterns of character covariation (Klingenberg and Spence, 1993). Although the resolution of allometric analysis in genetic variation among populations is believed to be inferior to shape analysis (Wiig, 1985), allometry is at least as heritable as shape (Atchley, 1983). The population structure of kuruma shrimp in these sampling areas was examined by shape comparison by Tzeng and Yeh (1999a) whose results were similar to ours, so the examination in genetic variation between populations by allometric comparison may provide extra information on population structure. However, because combinations of genetic and environmental factors modify growth processes (Cock, 1966) and allometric comparisons between groups using various statistical techniques often yielded different results (Leamy and Bradley, 1982; Gibson et al., 1984; Shea, 1985), some suggested that allometric patterns might not be solely used to infer the underlying heterochronic processes (Klingenberg and Spence, 1993; Tzeng et al., 1998). Pepin and Carr (1992) recommended that attempts to define population based on differences in morphometric characters must verified by genetic evidence in order to confirm that the former differences reflect some degree of reproductive isolation rather than simply environmental distinctiveness.

Differences between multivariate allometric coefficients obtained from two different groups were significant for each sex (Fig. 4). We found that FSL, FSW and FSH in females, but HSW, FSW and FSH in males were main characters to discriminate these two groups (Table 4). By comparing the allometric coefficients obtained from the second groups in each sex, we also found that the allometric difference between both sexes may mainly derive from ASW, HSW, and FSL characters (Table 4).

\section{Acknowledgements}

We would like to express the gratitude for funding supported by the Council of Agriculture, ROC, through contracts 85AST-1-1.13-FID-04. The authors would also like to express appreciation to our staff, the Demersal Research Center, Institute of Oceanography, National Taiwan University, for assistance in collecting samples.

\section{References}

Atchley, W.T., 1983. Some genetic aspects of morphometric variation. In: Felsenstein, J. (Ed.), Numerical Taxonomy. Springer, Berlin, pp. 346-363.

Bjorklund, M., 1993. Phenotypic variation of growth trajectories in finches. Evolution 7, 1506-1514.

Blackstone, N.W., 1987. Allometry and relative pattern and process in evolutionary studies. Syst. Zool. 36 (1), 76-78.

Boitard, M., Lefebvre, J., Sloignac, M., 1982. Analyse en composantes principales de la variabilite de taille, de croissance et de conformation desespeces du complexe Jaera albifrons (Crustaces isopodes). Cah. Biol. Mar. 23, 115-142.

Bookstein, F.L., Chernoff, B., Elder, R.L., Humphries, J.M., Smith, G.R., Strauss, R.E., 1985. Morphometrics in Evolutionary Biology: The Geometry of Size and Shape Change with Examples from Fishes, Special Publication No. 15. Academy of National Sciences, Philadelphia, 277 pp.

Cock, A.G., 1966. Genetical aspects of metrical growth and form in animals. Q. Rev. Biol. 41, 131-190.

Efron, B., Tibshirani, R., 1986. Bootstrap methods for standard errors, confidence intervals, and other measures of statistical accuracy. Stat. Sci. 1, 54-77.

Gibson, A.R., Baker, A.J., Moeed, A., 1984. Morphometric variation in introduced populations of the common myna (Acridotheres tristis): an application of the jackknife to principal component analysis. Syst. Zool. 33, 408-421.

Huxley, J.S., 1932. Problems of Relative Growth. Methuen, London.

Jolicoeur, P., 1963. The multivariate generalization of the allometry equation. Biometrics 19, 497-499.

Klingenberg, C.P., 1996. Multivariate allometry. In: Marcus, L.F., Corti, M., Loy, A., Naylor, G.J.P., Slice, D.E. (Eds.), Advances in Morphometrics. Plenum Press, New York, pp. 2350 .

Klingenberg, C.P., Spence, J.R., 1993. Heterochrony and allometry: lessons from the water strider genus Limnoporus. Evolution 47 (6), 1834-1853.

LaBarbera, M., 1989. Analyzing body size as a factor in ecology and evolution. Ann. Rev. Ecol. Syst. 20, 97-117.

Leamy, L., Bradley, D., 1982. Static and growth allometry of morphometric traits in randombred house mice. Evolution 36 (6), 1200-1212.

Liao, I.C., Chen, H.P., 1972. Taxonomic studies on the marine prawns at Tungkang area in Taiwan. I. On the four species of Penaeus: P. semisulcatus, $P$. japonicus and P. teraoi. Aquiculture 2 (1), 1-16.

Pepin, P., Carr, S.M., 1992. Morphometrical, meristic, and genetic analysis of stock structure in juvenile Atlantic cod (Gadus 
morhua) from the Newfoundland shelf. Can. J. Fish. Aquat. Sci. 50, 1924-1933.

Riska, B., 1981. Morphological variation in the horseshoe crab Limulus polyphemus. Evolution 35, 647-658.

Shea, B.T., 1985. Bivariate and multivariate growth allometry: statistical and biological considerations. J. Zool. (London) 206, 367-390.

Tsai, W.S., Chung, C.S., Chen, C.H., Lin, S.M., 1992. Preliminary study on the resources of kuruma prawn in the coastal waters of Penghu. Taiwan Fish. Res. 1 (1), 19-25.

Tzeng, T.D., Yeh, S.Y., 1999a. Analysis of the morphometric characters of kuruma shrimp (Penaeus japonicus) in the East
China Sea and the Taiwan Strait. J. Fish. Soc. Taiwan 26 (4), 203-212.

Tzeng, T.D., Yeh, S.Y., 1999b. Permutation tests for difference between two multivariate allometric patterns. Zool. Stud. 38 (1), $10-18$.

Tzeng, T.D., Chiu, C.S., Yeh, S.Y., 1998. Comparison of multivariate allometric coefficients in red-spot prawn (Metapenaeopsis barbata) from adjacent waters off Taiwan. J. Fish. Soc. Taiwan 25 (2), 85-92.

Wiig, Ø., 1985. Multivariate variation in feral American mink (Mustela vison) from Southern Norway. J. Zool. (London) 206, 441-452. 